\title{
Characterization of thiamine uptake and utilization in Candida spp. subjected to oxidative stress*
}

\author{
Natalia Wolak, Massimo Tomasi, Andrzej Kozik and Maria Rapala-Kozik® \\ Faculty of Biochemistry, Biophysics and Biotechnology, Jagiellonian University in Krakow, Kraków, Poland
}

Candida species are associated with an increasing number of life-threatening infections (candidiases), mainly due to the high resistance of these yeast-like fungi to antifungal drugs and oxidative stress. Recently, thiamine (vitamin B1) was found to alleviate stress responses in Saccharomyces cerevisiae; however, thiamine influence on defense systems in pathogenic fungi has never been investigated. The current work was aimed to elucidate the role of thiamine in stress reactions of $C$. albicans, C. glabrata, C. tropicalis and C. dubliniensis, subjected to hydrogen peroxide treatment. As compared to S. cerevisiae, Candida strains exposed to oxidative stress showed: (i) a much higher dependence on exogenous thiamine; (ii) an increased demand for thiamine diphosphate (TDP) and TDP-dependent enzyme, transketolase; (iii) no changes in gene expression of selected stress markers - superoxide dismutase and catalase - depending on thiamine availability in medium; (iv) a similar decrease of reactive oxygen species (ROS) generation in the presence of thiamine. Moreover, the addition of therapeutic doses of thiamine to yeast culture medium revealed differences in its accumulation between various Candida species. The current findings implicate that the protective action of thiamine observed in S. cerevisiae differs significantly form that in pathogenic Candida strains, both in terms of the cofactor functions of TDP and the effects on fungal defense systems.

Key words: vitamin B1, thiamine diphosphate-dependent enzymes, antioxidants, reactive oxygen species, Candida spp.

Received: 23 March, 2015; revised: 20 April, 2015; accepted: 14 May, 2015; available on-line: 18 August, 2015

\section{INTRODUCTION}

Candida albicans is the most prevalent infectious fungus, present in numerous niches as a part of normal microbiota within a human host. In healthy individuals, its occurrence may be completely unnoticed; however, under conditions of severe defects in immune defenses, C. albicans can cause life-threatening systemic infections (Molero et al.,1998; Dantas et al., 2015). In recent years, several non-albicans Candida species, e.g., C. glabrata and C. tropicalis, has been found to be associated with an increasing number of systemic candidiases, reaching a mortality rate of up to $40 \%$ (Pfaller \& Diekema, 2007). C. tropicalis is closely related to $C$. albicans and both organisms represent the 'CTG clade' species that possess a unique CUG codon for serine (Fig. 1) (Dujon et al., 2004). In contrast, C. glabrata is evolutionary more related to baker's yeast Saccharomyces cerevisiae, and thus, its adaptation as mammalian commensal developed indepen-

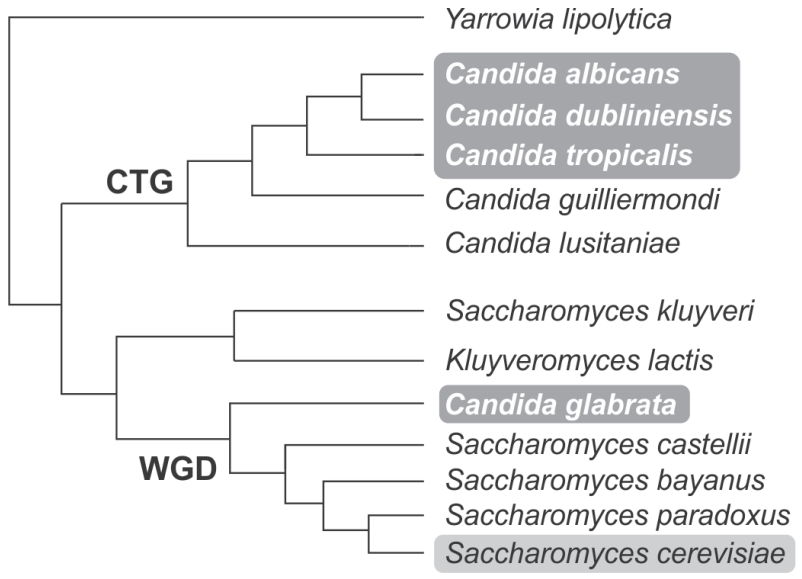

Figure 1. Phylogenetic tree of selected Saccharomycotina species.

CTG denotes the appearance of a new CUG codon for serine in selected Candida species, while WGD stands for the whole genome duplication that occurred in the lower branch of presented phylogenic tree. According to (Brown et al., 2014), modified.

dently from other Candida species (Roetzer et al., 2011b). In 1995, a new C. dubliniensis strain was isolated from HIV-infected individuals (Sullivan et al., 1995). Despite its extremely high similarity to C. albicans, allowing to differentiate the two species only with DNA fingerprinting methods, C. dubliniensis was identified in less than $3 \%$ of all candidiases and is associated mainly with benign superficial infections of vaginal and oral mucosa (Sullivan et al., 1995; Pfaller \& Diekema, 2007; Moran et al., 2012).

Integrated genomic and proteomic approaches are routinely applied to identify factors that contribute to C. albicans virulence and to investigate their occurrence in other Candida strains (Fernández-Arenas et al., 2007; Selmecki et al., 2010). In the light of the ongoing process of genome sequencing of the non-albicans species, the gene expression analyses using microarrays in selected strains at various stages of infection seems to be especially important (Fradin et al., 2003).

e-mail: maria.rapala-kozik@uj.edu.pl

*Preliminary report on the same subject has been presented during the 42nd Winter School of Faculty of Biochemistry, Biophysics and Biotechnology, Zakopane 10-14 February 2015

Abbreviations: CAT, catalase; $\mathrm{KGDH}$, a-ketoglutarate dehydrogenase complex; ROS, reactive oxygen species; SOD, superoxide dismutase; TA, thiamine; TDP, thiamine diphosphate; THI80, thiamine pyrophosphokinase; TKL, transketolase. 
The large differences in pathogenicity observed between various Candida species are shown to be strongly connected to the adaptations to stress conditions, and specific virulence factors utilized during the contact with host defense systems (d'Enfert, 2009). The latter include mainly adhesins, extracellular proteolytic enzymes and morphological changes (Naglik et al., 2003; Sudbery et al., 2004). The resistance of Candida species to oxidative stress is also of particular importance, as the main mechanism utilized by phagocytic cells - neutrophils and macrophages - to kill pathogens, involves the release of large amounts of reactive oxygen species (ROS) in the process known as oxidative burst (Babior et al., 1973; Wojtaszek, 1997).

The antioxidative mechanisms that are exploited by Candida cells to deal with stress conditions, include both enzymatic action of catalase, superoxide dismutases and different peroxidases and non-enzymatic protective molecules, such as glutathione and trehalose (Alvarez-Peral et al., 2002; Miramón et al., 2012; Dantas et al., 2015). Recently, thiamine (vitamin B1) has been found to ameliorate the effects of cellular stress in S. cerevisiae, through lowering the intracellular ROS level and reducing their deleterious effects on protein oxidation (Wolak et al., 2014). It is already known that despite the role of thiamine diphosphate (TDP) in basic cellular metabolism (Sauberlich, 1967; Bettendorff et al., 1996), thiamine can play other roles in stress responses in various organisms. In particular, thiamine can confer the resistance against oxidative agents in plants and bacteria Jung \& Kim, 2003; Tunc-Ozdemir et al., 2009; Rapala-Kozik et al., 2012) and, in forms of thiamine triphosphate and its adenylated derivatives, it can also serve as a signaling molecule under stress conditions (Lakaye et al., 2004; Gigliobianco et al., 2010). Although the mechanisms of the protective action of thiamine has not yet been recognized, the proposed thiamine oxidation upon the contact with free radicals can result in formation of thiamine thiols and tricyclic thiochrome derivatives (Lukienko et al., 2000; Stepuro et al., 2012).

A possible involvement of thiamine in stress responses of pathogenic Candida species has not been investigated previously. As the resistance of Candida cells to oxidants is much higher than that of $S$. cerevisiae (AlvarezPeral et al., 2002), it is particularly interesting if thiamine can affect the redox status in these fungal organisms, so specifically adapted to severe stress conditions. Thus, the main aim of this work was to analyze the oxidative stress response in several Candida species, in comparison to yeast $S$. cerevisiae, depending on the thiamine availability in culture medium.

\section{MATERIALS AND METHODS}

Materials. Culture media, YPD and Edinburgh Minimal Medium (EMM2), were obtained from Difco and US Biological respectively. Reagents for molecular biology experiments were obtained from Fermentas (GeneJet RNA Isolation Kit, dNTPs), Sigma (On-Column DNase, TRI Reagent), Promega (M-MLV Reverse Transcriptase) and KAPA (Universal SYBR Green Kit). All other reagents were purchased from Sigma.

Yeast strains and culturing. Saccharomyces cerevisiae BY4741 wild type strain was purchased from Euroscarf (Germany). Candida albicans ATCC 10231 strain was obtained from American Type Culture Collection (USA) and Candida dubliniensis NCYC 2670 was obtained from National Collection of Yeast Cultures (Great Britain).
Candida glabrata and Candida tropicalis strains were kindly provided by Dr Trojanowska (Jagiellonian University in Krakow, Poland).

Yeasts were grown in standard YPD medium or in a defined EMM2 medium, supplemented with vitaminfree casein hydrolysate $(20 \mathrm{mg} / \mathrm{ml})$, amino acids $(20 \mu \mathrm{g} /$ $\mathrm{ml}$ tryptophan and $40 \mu \mathrm{g} / \mathrm{ml}$ methionine, leucine and histidine), uracil $(120 \mu \mathrm{g} / \mathrm{ml})$ and vitamins $(0.4 \mu \mathrm{g} / \mathrm{ml}$ pyridoxine, niacin and pantothenic acid, $0.2 \mu \mathrm{g} / \mathrm{ml}$ riboflavin and $2 \mathrm{ng} / \mathrm{ml}$ biotin), at $30^{\circ} \mathrm{C}$ on orbital shaker (180 r.p.m.), until they reached an optimal growth phase $\left(\mathrm{OD}_{600}\right.$ value of $0.4-0.5$ for the gene expression analyses and of $0.8-1$ for the other assays). Stress conditions were established by transferring cell pellets into fresh medium with hydrogen peroxide for 1 hour. Unless stated otherwise, $S$. cerevisiae cells were treated with $1 \mathrm{mM} \mathrm{H}_{2} \mathrm{O}_{2}$ and Candida cells with $5 \mathrm{mM} \mathrm{H}_{2} \mathrm{O}_{2}$.

Measurement of growth rates. Yeasts were grown overnight in minimal EMM2 medium with or without thiamine and then diluted to final $\mathrm{OD}_{600}$ value 0.2 in fresh medium with hydrogen peroxide. After 1 hour of stress treatment, yeast cells were transferred to medium without the stressor and the further growth was monitored; for this monitoring, small amounts of cultures were withdrawn every 1 hour and the optical density was measured at $\lambda=600 \mathrm{~nm}$.

RNA isolation and quantitative PCR. Yeast cells were distupted with glass beads (425-600 $\mu \mathrm{m}$, Sigma) and TRI Reagent using FastPrep Instrument $(6.0 \mathrm{~m} / \mathrm{s}$, 45 seconds). Due to the stronger cell wall, the disruption for Candida cells was performed in two 45-second cycles. Total RNA was isolated using GeneJet RNA Isolation Kit with DNase treatment and the quality of RNA was assessed by separation in agarose gel under denaturing conditions. First strand cDNA was synthesized using $2 \mu \mathrm{g}$ of total RNA and dT18 primers with M-MLV Reverse Transcriptase, and subsequently diluted two fold with water. Real Time PCR was performed on Step One Instrument (Applied Biosystems) with SYBR Green for fluorescent labeling, in a final volume of $10 \mu \mathrm{l}$. The applied pairs of gene-specific primers (Genomed) are listed in Table 1 . The reaction conditions were: $95^{\circ} \mathrm{C}$ for 10 min, followed by 40 cycles of $94^{\circ} \mathrm{C}$ for $15 \mathrm{~s}, 57^{\circ} \mathrm{C}$ for $15 \mathrm{~s}$, and $72^{\circ} \mathrm{C}$ for $20 \mathrm{~s}$. The RDN18 and ACT1 genes were used as references as they showed the most stable expression under stress conditions. Appropriate negative controls with RNA or water instead of cDNA were also used. Relative fold changes in expression levels were calculated using the $2^{-\triangle \Delta C T}$ method (Livak \& Schmittgen, 2001).

Intracellular thiamine and TDP levels. Yeast cells were disrupted as for RNA isolation in the presence of $12 \%$ trichloroacetic acid (TCA) that was then removed with ether extraction. Samples were analyzed using reverse-phase high pressure liquid chromatography (RPHPLC) on SUPELCOSIL ${ }^{\mathrm{TM}}$ LC-18 column (Sigma), with a post-column derivatization using $90 \mu \mathrm{M}$ sodium hexacyanoferrate in $0.56 \mathrm{M} \mathrm{NaOH}$. The fluorescence was monitored at $365 \mathrm{~nm}$ and $430 \mathrm{~nm}$ excitation and emission wavelengths (Rapala-Kozik et al., 2008). For RP-HPLC separation, a gradient elution (0-98\% B, 16 min) was used, (solvent A: $15 \mathrm{mM}$ ammonium citrate (pH 4.2); solvent B: $0.1 \mathrm{M}$ formic acid with $55 \mathrm{mM}$ diethylamine).

Detection of reactive oxygen species. The ROS levels were determined with dihydroethidium (DHE) fluorescent dye (Fink et al., 2004). Yeast cells were grown overnight in the presence of thiamine $(1.4 \mu \mathrm{M})$ or its absence, then washed twice with phosphate-buffered saline 
Table 1. List of primers used in this study.

\begin{tabular}{|c|c|c|c|}
\hline Gene & Forward & Reverse & Strain \\
\hline CTT1 & GTCCATACTCCAAAGGTGATT & TACTTCGTCGTTGTCTTCATT & S. cerevisiae \\
\hline KGD1 & GGAAGCAACGCTCTGGTTTA & TCTTGGGTCTTCATTGGCTAGT & S. cerevisiae \\
\hline RDN18 & CGGCTACCACATCCAAGGAA & GCTGGAATTACCGCGGCT & S. cerevisiae \\
\hline SOD2 & TCACAAACCACTGTCTATTCTGG & GACTGCCAAACTGCTCGTC & S. cerevisiae \\
\hline THI80 & AGAACTAATCCATCCAAACG & TCAAGTCATGCAGCTTCC & S. cerevisiae \\
\hline TKL1 & AGCCCTTGACTTCCAACC & ATAGCGTGTTCTCTAATACCG & S. cerevisiae \\
\hline ACT1 & GATTTTGTCTGAACGTGGTAACAG & GAGTTGAAAGTGGTTTGGTCAATAC & C. albicans \\
\hline CAT1 & GATTCTCTACTGTTGGTGGTG & GTGAGTTCTGGGTTTCTCTT & C. albicans \\
\hline KGD1 & TTAGAGTGTCGGGTCAAGAT & CTGGGGAGGTCAAGGAGTA & C. albicans \\
\hline SOD2 & CGTTGAAGCCAAATCTAAAG & GAGAGACAGGAGCCAAGTIT & C. albicans \\
\hline THI80 & АTCTCTCCACCTTCAGACTCAT & ACTATTTGCTCCACCATCG & C. albicans \\
\hline TKL1 & TCAAGAAAGACAACCCAGAC & GCAAGGAAACAACATTAGCC & C. albicans \\
\hline ACT1 & CCTCCAGAAAGAAAATACTCTG & TTGTGATGAACAATAGATGGAC & C. dubliniensis \\
\hline CAT & GTTITGGGATTACTTGACTAGC & TAAGAAGCTGGAGTACCTCTGT & C. dubliniensis \\
\hline $\mathrm{KGDH}$ & GCTTACCAAGTTAGAGGTCATC & TACTCTTCTTACCACCTTGAGC & C. dubliniensis \\
\hline SOD & GAGCTAAATACTCCGCTAGAAA & GGGTATAGACGTTGTCAGTTIT & C. dubliniensis \\
\hline THI80 & TAATTTCTCCACCTTCAGACTT & TGGTTTGTGATGTAGTTGTCTT & C. dubliniensis \\
\hline TKL & AATGTCCCAGAAAGATACAGAG & CAAAGTTGGTTIATCGGTAGAT & C. dubliniensis \\
\hline ACT1 & GAGGTATTITGACTTTGCGTTA & GTGTTCTTCTGGGGCGACT & C. glabrata \\
\hline CTA1 & GCGTAGAGTCGGTAAGATGGTC & GACAGGGATTTGGTGGAAGTTA & C. glabrata \\
\hline $\mathrm{KGDH}$ & CGTAAACCAAACGAATCCATCT & TCTACCAAGGACAACAGGGTCT & C. glabrata \\
\hline SOD & TAGAGTGGGACTTCGGTGCT & ATAGGTCTGGTGGTGCTTGG & C. glabrata \\
\hline THI80 & CTGTTGCCTGTTGGTCTTCC & GCTGCTCACTCGTCCAGATT & C. glabrata \\
\hline TKL1 & ATCGTCTTCCAAAGCATCTACG & CATACCGTGTTCTCTGATACCG & C. glabrata \\
\hline ACT1 & ATACTCTGTCTGGATCGGTGGT & TTTGTGGTGGACAATAGATGGA & C. tropicalis \\
\hline CAT & GATTGATTCCTTGGCTCATTTC & AACACCATAAGCACCAGAACCT & C. tropicalis \\
\hline $\mathrm{KGDH}$ & AAGAGATTTGGTTTGGAAGGTG & AGATGGATTCGTTTGGTTTACG & C. tropicalis \\
\hline SOD & CAATGTTACCCAAGTCACCAAC & CTGAATCCGAACCAACCACTAT & C. tropicalis \\
\hline THI80 & ATCCTTGTTITGCGATTCTGG & СACCATCTGTGTTGTCCATTCT & C. tropicalis \\
\hline TKL & ATTTCCAACCACCATCTACTGG & AATAGCACCCATACCGTGTTCT & C. tropicalis \\
\hline
\end{tabular}

(PBS) and resuspended in PBS with $25 \mu \mathrm{g} / \mathrm{ml} \mathrm{DHE}$ to final $\mathrm{OD}_{600}=0.8$ and incubated for 10 minutes at $30^{\circ} \mathrm{C}$ in the dark.

After washing twice with PBS, the fluorescence signal in the sample was detected in the presence of $0-25 \mathrm{mM}$ $\mathrm{H}_{2} \mathrm{O}_{2}$, using a BioTek SYNERGY H1 microplate reader $(\lambda$ exc $=520$ and $\lambda \mathrm{em}=610 \mathrm{~nm})$.

Determination of protein concentration. Protein concentration was measured by the Lowry method (Lowry et al., 1951).

Statistical analysis. All experiments were repeated at least 3 times to ensure proper analysis of statistical significance ( $t$-test, $P<0.05)$.

\section{RESULTS}

Candida spp. are present in numerous niches within the human host, that significantly differ in terms of thiamine availability. A particularly high amount of thiamine compounds was found in colon and skin, in comparison to a rather low concentration in vaginal mucosa (Gangolf et al., 2010). Moreover, thiamine is known to be used in large doses for treatment of several pathological conditions, such as thiamine-responsive megaloblastic anemia (TRMA), Alzheimer disease or diabetes (Ozdemir et al., 2002; Thornalley, 2005; Gibson \& Blass, 2007), resulting in much higher local concentrations of thiamine than 


\section{S. cerevisiae}

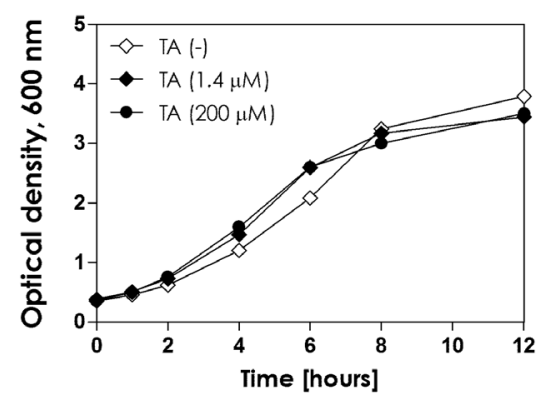

C. albicans

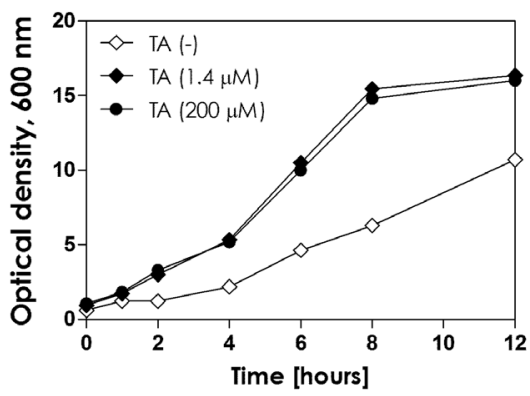

C. dubliniensis

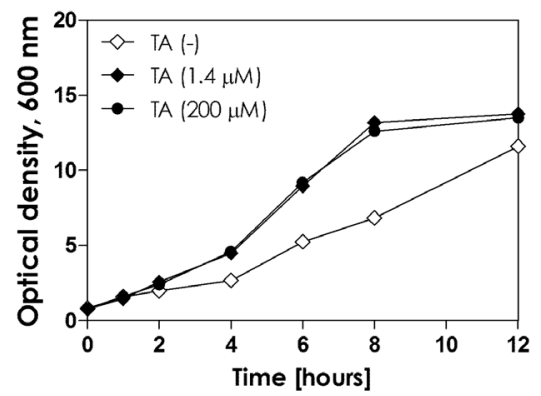

C. glabrata

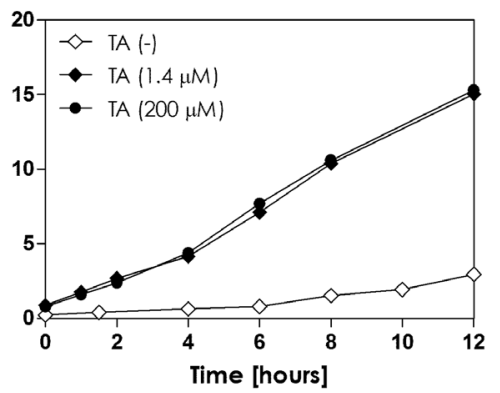

C. tropicalis

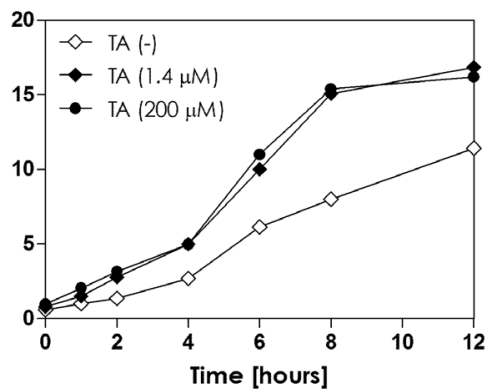

Figure 2. The growth of baker's yeast and selected Candida species, depending on thiamine availability in culture medium.

Yeasts were grown overnight in minimal EMM2 medium with or without thiamine and then were diluted to final $\mathrm{OD}_{600}$ value 0.2 . The growth rate was then monitored at $\lambda=600 \mathrm{~nm}$. Error bars represent the standard deviation.

under physiological state. In order to determine how different thiamine availability can affect Candida cells, three thiamine concentrations were selected, $0,1.4 \mu \mathrm{M}$ and $200 \mu \mathrm{M}$. The $1.4 \mu \mathrm{M}$ concentration represents the level of thiamine in the YPD medium, that is used for optimal yeast propagation, whereas the $200 \mu \mathrm{M}$ concentration corresponds to conditions during the medical treatments.

\section{Effects of exogenous thiamine on the growth of selected Candida strains and the intracellular thiamine accumulation}

A significant growth impairment of Candida cultures in the absence of thiamine was observed, especially in the case of $C$. glabrata (Fig. 2). The differences in growth rates between $\mathrm{TA}(-)$ and $\mathrm{TA}(+)$ conditions were much higher than in $S$. cerevisiae cultures, suggesting a lower rate of thiamine biosynthesis in Candida cells. The results correlated with intracellular thiamine accumulation, as no thiamine was detected in case of all selected Candida strains (Fig. 3). Probably, all thiamine synthesized under these conditions was already transformed to the active form (TDP), that could be detected at a similar level in $S$. cerevisiae. Only in the case of C. glabrata, no TDP was found in thiamine deficient medium, the finding that explains the impaired growth of this species under these conditions.

The addition of larger amounts of thiamine to culture medium did not affect the growth of $S$. cerevisiae or Candida spp., suggesting that the $1.4 \mu \mathrm{M}$ concentration is high enough to ensure optimal growth of yeasts. However, the supplementation of medium with $200 \mu \mathrm{M}$ thiamine revealed differences in intracellular thiamine accumulation between analyzed strains (Fig. 3). Under these conditions, both $S$. cerevisiae and $C$. tropicalis showed at least 8-times higher amount of thiamine than other species, suggesting that they possess mechanisms of a much tighter control of thiamine uptake. The differences in thiamine uptake regulation may be a part of the adaptation to the conditions within the host, and in this aspect C. tropicalis seems to have diverged from other Candida strains.

The analyses of gene expression of thiamine pyrophosphokinase (THI80), that converts thiamine into TDP, revealed its 6-fold upregulation in C. tropicalis when compared to other strains under TA(-) conditions (Fig. 4). The results may indicate a higher importance of the role of TDP-dependent enzymes in this 
TDP

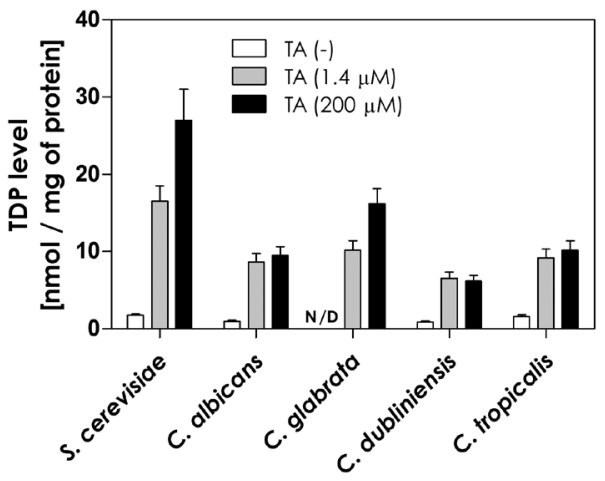

THIAMINE

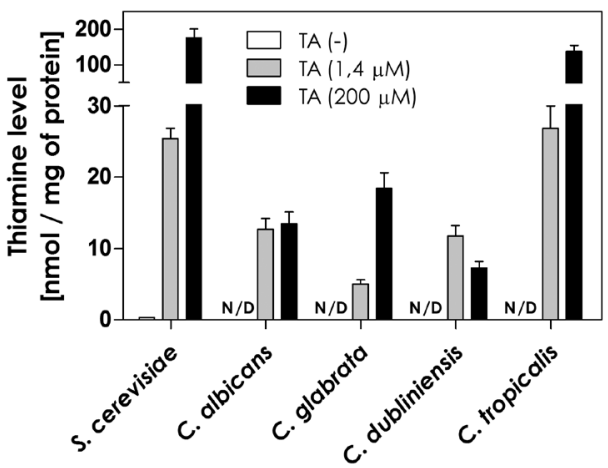

Figure 3. The intracellular accumulation of thiamine and TDP in S. cerevisiae and selected Candida species, depending on thiamine availability in culture medium.

Yeasts were grown overnight in minimal EMM2 medium with or without thiamine and then transferred to fresh medium for additional 1 hour. Thiamine compounds were extracted with $12 \%$ TCA and quantified using RP-HPLC method with post-column derivatization and fluorometric detection. N/D - thiamine or TDP not detected.

\section{THI80}

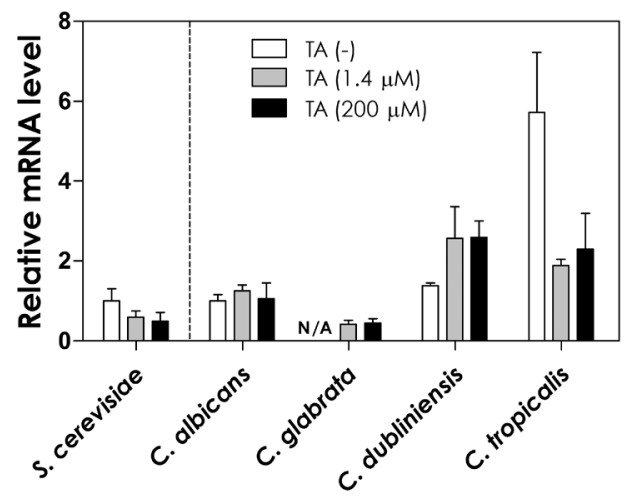

KGDH

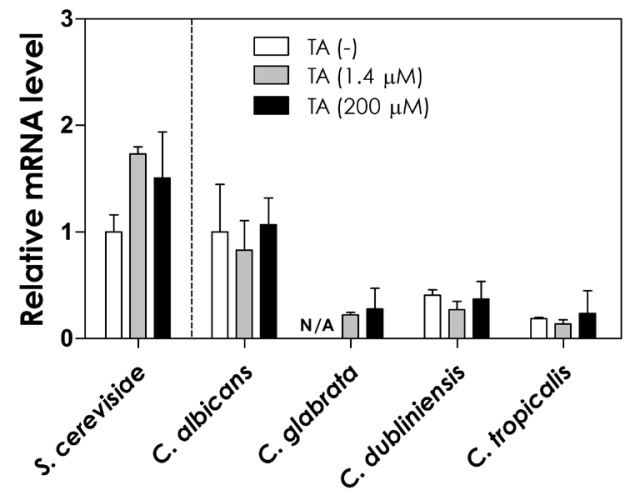

TKL

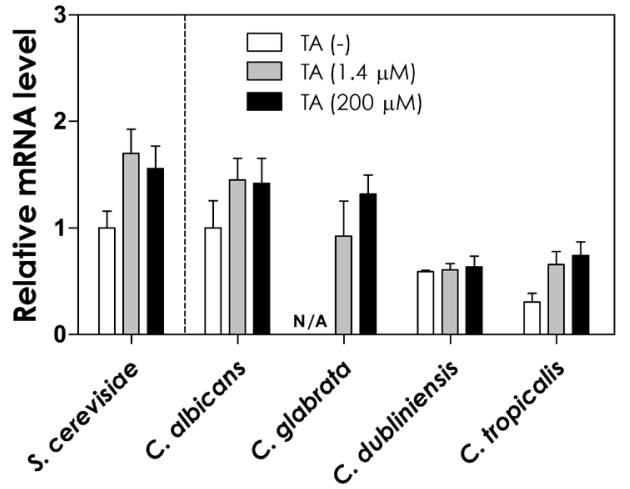

Figure 4. The expression of genes coding for thiamine pyrophosphokinase and major TDP-dependent enzymes - transketolase (TKL) and a-ketoglutarate dehydrogenase (KGDH) — in baker's yeast and selected Candida species, depending on thiamine availability in growth medium.

The gene expression analysis was performed using Real Time PCR with SYBR Green for fluorescent labeling and ACT1/RDN18 genes as references. The results for all Candida strains were calculated versus $C$. albicans TA(-) samples (equal 1); a separate reference value was made for S. cerevisiae cells. Error bars represent the standard deviations calculated according to Livak \& Schmittgen, 2001 . N/A — sample not available due to very poor growth of C. glabrata culture in the absence of thiamine in medium.

Candida strain. Indeed, 2-fold higher expression of transketolase gene (TKL) was observed in C. tropicalis cells in the presence of thiamine in the medium. Intuitively, one could expect that the higher expression should be associated with thiamine deficiency; however, the same dependence was recently observed in S. cerevisiae
(Wolak et al., 2014) and in previous analyses of human cells and brain sections (Pekovich et al., 1998; Shi et al., 2008). The other Candida species did not reveal any significant differences in expression of THI8O and main TDP-dependent enzymes, e.g., transketolase (TKL) and $\alpha$-ketoglutarate dehydrogenase $(K G D H)$. 


\section{TA (-) CONDITIONS}

\section{TA (+) CONDITIONS}

\section{S. cerevisiae}
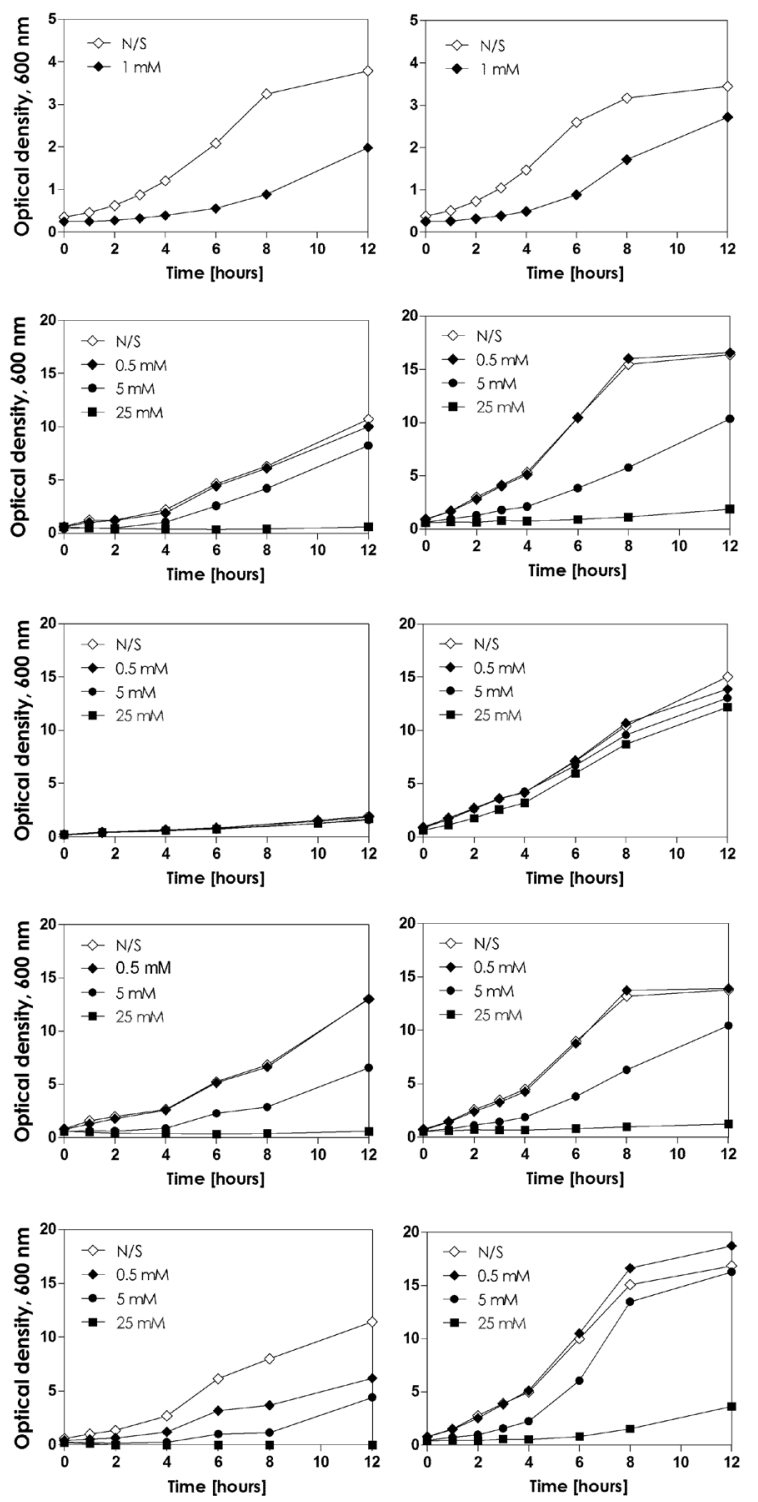

Figure 5. The growth of baker's yeast and selected Candida species under conditions of oxidative stress, depending on thiamine availability in culture medium.

Yeasts were grown overnight in minimal EMM2 medium with or without thiamine and then transferred to fresh medium with hydrogen peroxide. After 1 hour of treatment, the further growth in a new portion of medium without the stressor was monitored at $\lambda=600 \mathrm{~nm}$. Error bars represent the standard deviation. N/S - non-stressed control.

\section{An increased demand for thiamine and TDP in Candida cells under oxidative stress}

Candida species are known to be much more resistant to oxidants than yeast $S$. cerevisiae, being able to survive short treatment of hydrogen peroxide at up to $50 \mathrm{mM}$ concentrations (Alvarez-Peral et al., 2002). In order to compare the stress responses of those organisms, their viability at different concentrations of hydrogen peroxide was tested (Fig. 5).

For further studies, $1 \mathrm{mM}$ and $5 \mathrm{mM} \mathrm{H}_{2} \mathrm{O}_{2}$ were chosen for $S$. cerevisiae and Candida species, respectively, as both concentrations caused comparable survival of the cells.
The same analysis showed that under conditions of thiamine availability in the medium, C. glabrata cells showed the highest resistance to hydrogen peroxide, with almost no growth inhibition even when subjected to 25 $\mathrm{mM}$ stressor. Increased survival under stress and ability to growth at $25 \mathrm{mM} \mathrm{H} \mathrm{H}_{2} \mathrm{O}_{2}$ was observed also for $C$. tropicalis. Interestingly, both species presented also the largest growth defects under stress when relied completely on thiamine biosynthesis, showing the negative correlation between stress resistance and ability to synthesize thiamine.

The same species, C. glabrata and C. tropicalis accumulated more thiamine when subjected to stress treatments (Fig. 6). When thiamine was not present in the medium, thiamine again was not detected, whereas TDP level was unchanged, apart from $C$. dubliniensis (almost 2-fold less TDP under stress). The results were correlated with a very high expression of THI8O gene in C. dubliniensis (more than 7-fold), that was probably aimed to compensate the small TDP level (Fig. 7). The high expression of thiamine pyrophosphokinase was also observed in $C$. tropicalis cells (more than 2-fold), possibly supporting the previous hypothesis on the increased importance of the TDPdependent enzymes in this Candida strain under stress conditions.

An important difference between S. cerevisiae and Candida spp. was found in the transketolase gene expression. While in S. cerevisiae the TKL1 gene is strongly repressed after hydrogen peroxide treatment as a part of the general metabolic shutdown (Gasch et al., 2000; Ralser et al., 2007), in all Candida cells transketolase was upregulated. Although it is known that in $S$. cerevisiae cells the activity of transketolase is largely controlled metabolically (Ralser et al., 2009), its higher activity was already shown under oxidative stress (Kowalska et al., 2012), as well as for C. glabrata and under heavy metal treatment for C. albicans (Yin et al., 2009; Seneviratne et al., 2010).

\section{Changes in defense systems of Candida strains under oxidative stress, depending on thiamine availability}

In order to determine the protective effect of thiamine on Candida cells, the expression of two main stress markers, superoxide dismutase $(S O D)$ and catalase $(C A T)$, depending on thiamine availability in medium was measured (Fig. 8). In $S$. cerevisiae cells, the addi- 


\section{TA (-) CONDITIONS}

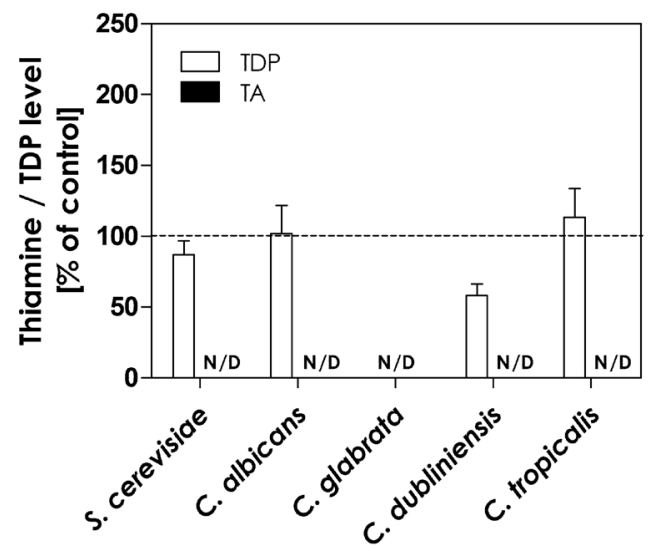

TA (+) CONDITIONS

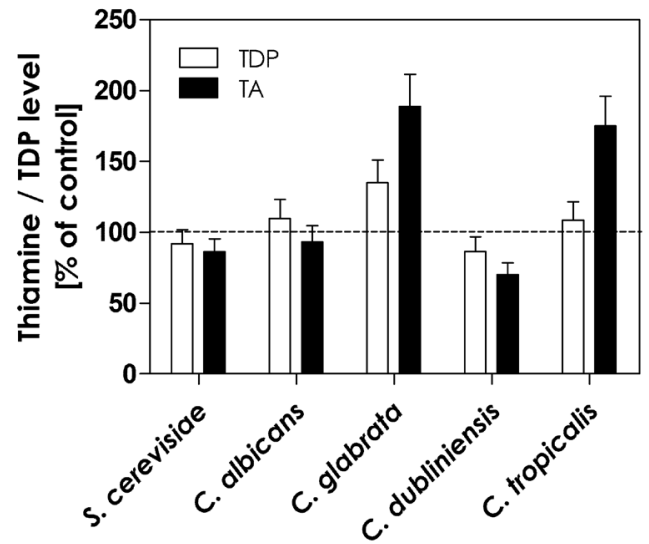

Figure 6. The intracellular accumulation of thiamine and TDP in S. cerevisiae and selected Candida species subjected to oxidative stress, depending on thiamine availability in culture medium.

Yeasts were grown overnight in minimal EMM2 medium with or without thiamine and then transferred to fresh medium with hydrogen peroxide for 1 hour. Thiamine compounds were extracted with 12\% TCA and quantified using RP-HPLC method with post-column derivatization and fluorometric detection. N/D - thiamine or TDP not detected.

\section{THI80}

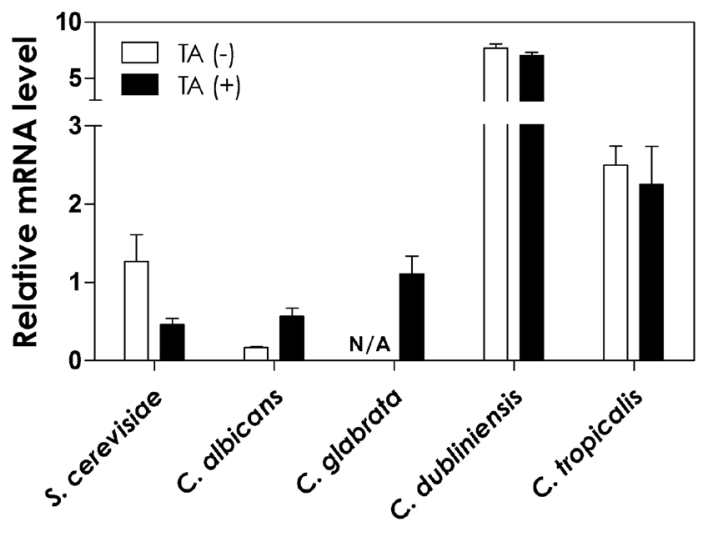

KGDH

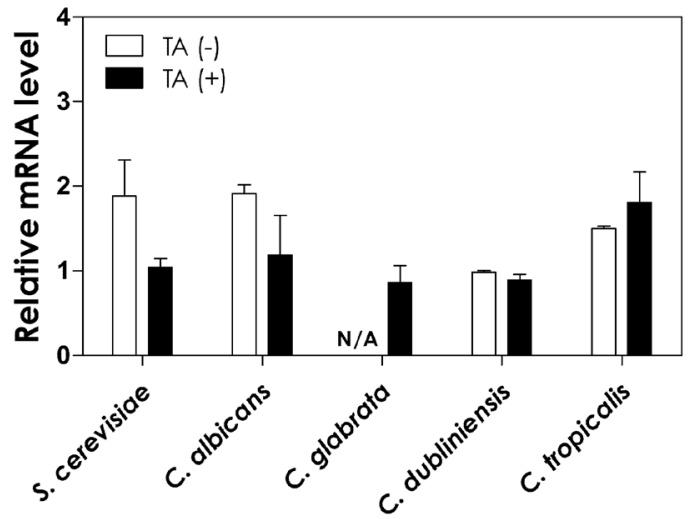

TKL

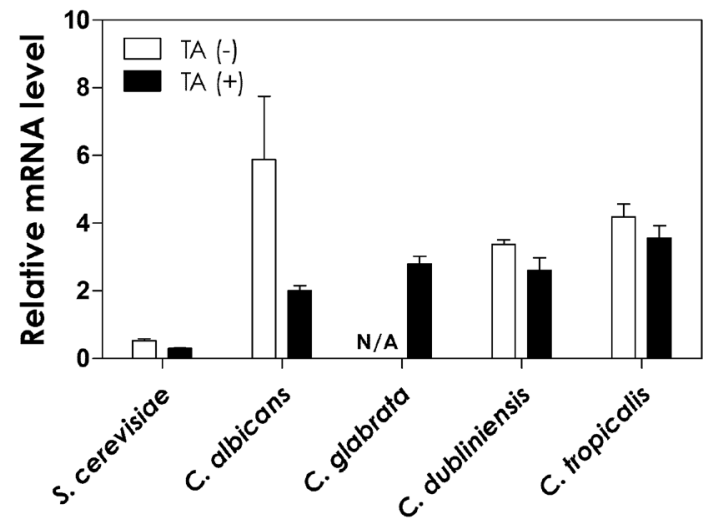

Figure 7. The expression of genes coding for thiamine pyrophosphokinase and major TDP-dependent enzymes - transketolase $(T K L)$ and a-ketoglutarate dehydrogenase $(K G D H)$ - in baker's yeast and selected Candida species under oxidative stress conditions, depending on thiamine availability in growth medium.

Yeasts were grown overnight in minimal EMM2 medium with or without thiamine and then transferred to fresh medium with hydrogen peroxide for 1 hour. The gene expression analyses were performed as described on Fig. 4 . The results represent relative gene expression compared to the control conditions (equal 1) for each strain separately. Error bars represent the standard deviations calculated according to Livak \& Schmittgen, 2001. N/A - sample not available due to very poor growth of C. glabrata culture in the absence of thiamine in medium. 
SOD

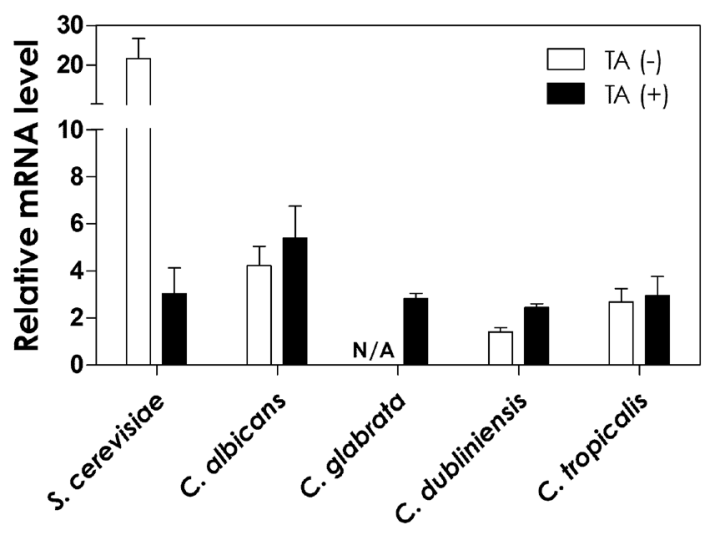

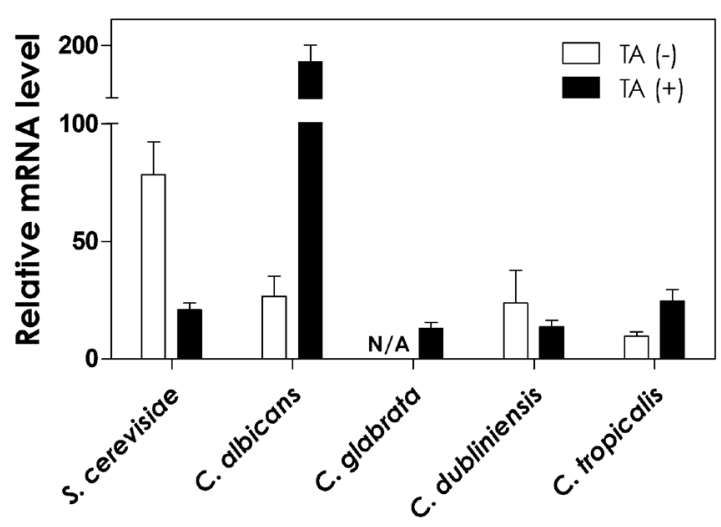

Figure 8. The expression of genes coding for major stress markers, superoxide dismutase (SOD) and catalase (CAT), in baker's yeast and selected Candida species under oxidative stress conditions, depending on thiamine availability in growth medium.

Yeasts were grown overnight in minimal EMM2 medium with or without thiamine and then transferred to fresh medium with hydrogen peroxide for 1 hour. The gene expression analyses were performed as described on Fig. 4. Error bars represent the standard deviations calculated according to Livak \& Schmittgen, 2001. N/A - sample not available due to very poor growth of C. glabrata culture in the absence of thiamine in medium.

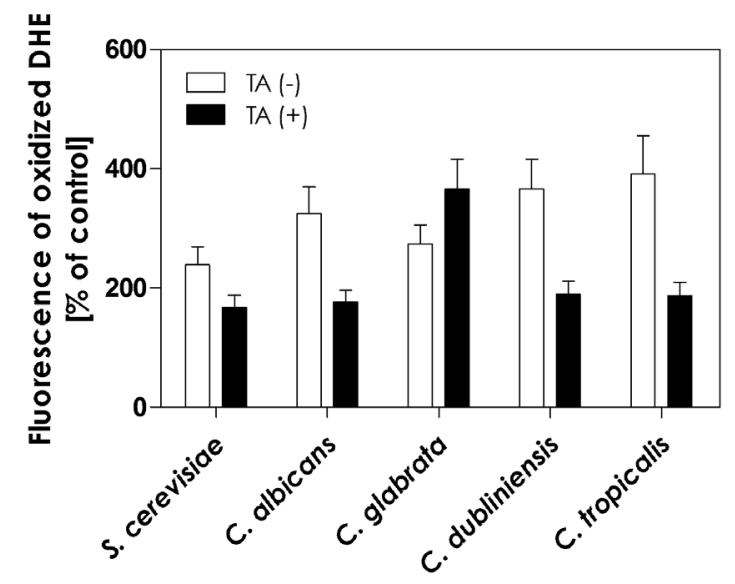

Figure 9. Reactive oxygen species (ROS) generation in S. cerevisiae and selected Candida strains, subjected to oxidative stress treatment, depending on thiamine availability in growth medium.

Yeasts were grown overnight in minimal EMM2 medium with or without thiamine. The ROS level was measured using $25 \mu \mathrm{g} / \mathrm{ml} \mathrm{di-}$ hydroethidium and the fluorescence signal was measured in the presence of hydrogen peroxide $(\lambda e x c=520 \mathrm{~nm}, \lambda e m=610 \mathrm{~nm})$.

tion of thiamine lowered the expression of both markers, suggesting its positive effect on the cellular redox state. This effect was not observed in any of the Candida strains tested, with even higher expression of SOD and $C A T$ when thiamine was present in culture medium, especially in the case of catalase gene in C. albicans cells (up to 10 -fold). The results may point at differential effects of thiamine on benign and pathogenic fungi under stress conditions. Most of Candida strains differ from S. cerevisiae with a number of isoforms of superoxide dismutase and catalase and their intracellular localization (Wysong et al., 1998; Frohner et al., 2009), a factor that could also affect the results.

However, most of Candida strains, similarly to S. cerevisiae cells, showed decreased ROS level when thiamine was present in culture medium (Fig. 9). Thus, thiamine can probably perform a protective action on Candida cells but the detailed mechanism may not be so straight- forward as in $S$. cerevisiae. The only strain that revealed an opposite effect in ROS production was $C$. glabrata but this could be due to the very poor growth of this fungus under TA $(-)$ conditions. Nevertheless, when thiamine was present in the medium, C. glabrata cells showed 2 -fold higher level of ROS than other species. This effect may be associated with their highest resistance to oxidative stress and ability to survive for a long time inside phagosomes, where C. glabrata cells are exposed to large doses of reactive species (Roetzer et al., 2010; Seider et al., 2014).

\section{DISCUSSION}

Candida yeasts are the major causative agents of invasive fungal infections among hospitalized patients, with non-albicans strains representing a significant number of total isolates (Krcmery \& Barnes, 2002; Zaoutis et al., 2005). In many of them, the process of the adaptation to human host environment developed differently, resulting in several specific traits, associated mainly with the virulence level. One of them is the ability to survive under oxidative stress conditions, commonly encountered during the contact with host defense system or with other microorganisms inhabiting the same niches in human body (Dantas et al., 2015). It is already known that thiamine can increase cell survival upon hydrogen peroxide treatment in baker's yeast $S$. cerevisiae, closely related to C. glabrata, mainly by reducing the intracellular ROS level and alleviating their negative effects on protein oxidation (Wolak et al., 2014). No study, however, was conducted in this regard to pathogenic Candida species.

Thiamine is an essential compound for all living organisms but only plants, microorganisms and some fungi are capable of its biosynthesis (Begley et al., 1999; Goyer, 2010). The synthesis of one thiamine molecule in yeast requires the equivalent of at least five adenosine5-triphosphate molecules; thus, when thiamine can be taken up from the external environment, the biosynthesis process is almost completely blocked (Iwashima \& Nose, 1976; Mojzita \& Hohmann, 2006). The thiamine biosynthesis pathway is well recognized in $S$. cerevisiae (Nosaka, 2006; Kowalska \& Kozik, 2008) but little is 
known about this process in Candida species. Recently, some homologues of the thiamine biosynthetic enzymes of $S$. cerevisiae have been characterized in C. albicans and C. glabrata (Paul et al., 2010; Lai et al., 2012). However, our analyses of Candida growth under different thiamine concentration in the medium revealed the significant growth impairment when thiamine was not present, in comparison to $S$. cerevisiae cells, indicating that thiamine biosynthesis is much less effective in Candida species. The results correlated with the intracellular levels of thiamine, that in all strains were under detection limits. In C. glabrata cells, no TDP could also be detected, explaining almost complete growth inhibition of this strain under TA $(-)$ conditions. The high dependence on the thiamine availability in host environment can be seen as a part of the adaptation to the commensal lifestyle of Candida species. Indeed, the loss of several genes involved in important cellular pathways, such as the metabolism of galactose (GAL1/7/10), phosphate (PHO3/5/11/12), nitrogen $(D A L 1 / 2)$ or sulphur (SAM4) was already observed in C. glabrata. Additionally, C. glabrata cells have lost the ability to synthesize some vitamins, such as niacin and pyridoxine (Kaur et al., 2005). Although the thiamine biosynthesis rate seem to be very low, the main protein involved in this process, Thi6, has been recently characterized (Paul et al., 2010), indicating that the thiamine biosynthetic ability may not be lost completely. The reduction in metabolic pathways was not observed in other Candida species, the feature that can be associated with a rather large evolutionary distance between C. glabrata and other Candida spp.. The differences, while comparing to $C$. albicans, may be also related to the ability to penetrate tissues by various Candida species (Fradin et al., 2003). C. albicans is able to form hyphae and actively migrate between different niches in the host organism (d'Enfert, 2009), where they can experience various thiamine availability. C. glabrata cells do not change their morphological form and localize mainly in the mucosa (Roetzer et al., 2011b), where main nutrients should be provided.

The addition of high doses of thiamine $(200 \mu \mathrm{M})$ to culture medium did not affect the growth of neither Candida nor Saccharomyces strains, however, it revealed differences in thiamine accumulation. In comparison to $S$. cerevisiae and $C$. tropicalis, that accumulated larger amounts of thiamine, other species seemed to regulate the uptake process much more tightly. However, the specific carrier for thiamine has been identified only in S. cerevisiae (Enjo et al., 1997; Singleton, 1997) and C. glabrata (Candida Genome Database, unpublished data). Both characterized proteins show very high similarity of amino acid sequences but our search for their homologues in other Candida strains was not successful.

The main part of our current work was devoted to the thiamine action in Candida cells under conditions of oxidative stress. The positive correlation between the resistance to hydrogen peroxide and to thiamine supplementation was observed. The low viability under TA(-) conditions is directly connected to previously described decreased ability to synthesize thiamine. Apart from C. glabrata, also C. tropicalis showed much more impaired growth under those conditions, suggesting that it can currently undergo the same process of metabolic reduction that was observed in C. glabrata. However, the identification of homologous proteins requires better characterization of genomes of many non-albicans Candida species.

The functions of thiamine are associated largely with the cofactor role of TDP in basic cellular metabolism.
The higher mRNA level of thiamine pyrophosphokinase, enzyme that activate thiamine to TDP, suggests that the cofactor function may be more important in Candida than Saccharomyces. Indeed, the expression of transketolase under oxidative stress was also upregulated in all Candida species, in contrast to its downregulation in Saccharomyces. An increased activation of transketolase upon hydrogen peroxide treatment was previously shown in C. glabrata (Seneviratne et al., 2010) and after cadmium treatment in C. albicans (Yin et al., 2009). The difference between Saccharomyces and Candida in transketolase gene expression may be associated with the higher dependence of the latter species on the oxygen metabolism and, thus, higher generation of oxygen radicals (Vázquez- Torres \& Balish, 1997). In Saccharomyces, the presence of even low amounts of ROS results in a temporary inhibition of Krebs cycle and redirection of metabolism to pentose phosphate pathway (Ralser et al., 2007).

The analyses of gene expression for selected stress markers revealed, that their downregulation in the presence of thiamine in S. cerevisiae was not observed in Candida cells. This finding may be interpreted in terms of different isoenzymes, expressed between both genera. C. albicans is equipped with five superoxide dismutases genes, that allow to expose the encoded Sod proteins on the yeast cell surface (Martchenko et al., 2004; Roetzer et al., 2011b). C. glabrata has two SOD genes, similarly to $S$. cerevisiae; however, their expression is differently regulated rendering the ability to survive at severe oxidative stress. Adaptation to host environment resulted also in reduction of catalase genes. C. albicans and C. glabrata appear to have only one catalase but it combines the different transcriptional regulation and different intracellular localization (Roetzer et al., 2010).

Despite the results obtained with stress markers, the protective effects of thiamine in Candida cells were confirmed by the observation of diminished generation of ROS in the presence of thiamine. An adverse response was observed only for C. glabrata, which was associated with poor growth of this yeast in the absence of thiamine and partially could result from their exceptional resistance to stress conditions (Roetzer et al., 2011a). It should be mentioned that Candida species developed different strategies to survive on contact with phagocytic cells, neutrophils and macrophages. Some of them, like C. albicans, can escape from phagosomes, whereas C. glabrata can survive for longer time inside phagosomes (Ferrari et al., 2011). This is mainly owing to ability of this species to actively suppress ROS production by neutrophils and relative resistance to starvation (Wellington et al., 2009).

Thus, our current findings implicate that the protective action of thiamine, observed in $S$. cerevisiae, differ significantly in pathogenic Candida species, both in terms of thiamine cofactor functions and the thiamine effects on defense systems.

\section{CONCLUSIONS}

Despite evolutionary differences between Candida strains, their adaptation to human host resulted in developing similar traits, allowing them for an effective invasion under conditions of weakened immune defenses. One of them is an increased resistance to oxidative stress but the role of thiamine in this process is less clear than in the stress reaction of $S$. cerevisiae. The more detailed analyses of defense system in Candida would require the use of other isoforms of stress markers, which diverged slightly from those observed in Sacharomyces ge- 
nus. Nevertheless, the reduced amount of oxygen species observed in the presence of thiamine confirmed at least its partial protective effect on Candida species.

\section{Acknowledgements}

This work was supported in part by the National Science Centre, Poland (based on the decision No. DEC$-2011 / 03 / \mathrm{N} / \mathrm{NZ1} / 01305$ to NW). The authors declare no conflict of interest.

\section{REFERENCES}

Alvarez-Peral FJ, Zaragoza O, Pedren Y (2002) Protective role of trehalose during severe oxidative stress caused by hydrogen peroxide and the adaptive oxidative stress response in Candida albicans. 148: 2599-2606.

Babior BM, Kipnes RS, Curnutte JT (1973) Biological defense mechanisms. The production by leukocytes of superoxide, a potential bactericidal agent. J Clin Invest 52: 741-744.

Begley TP, Downs DM, Ealick SE, McLafferty FW, Van Loon AP, Taylor S, Campobasso N, Chiu HJ, Kinsland C, Reddick JJ, Xi J (1999) Thiamin biosynthesis in prokaryotes. Arch Microbiol 171: 293300 .

Bettendorff L, Mastrogiacomo F, Kish SJ, Grisar T (1996) Thiamine, thiamine phosphates, and their metabolizing enzymes in human brain. J Neurochem 66: 250-258.

Brown AJP, Brown GD, Netea MG, Gow N (2014) Metabolism impacts upon Candida immunogenicity and pathogenicity at multiple levels. Trends Microbiol 22: 614-622.

d'Enfert C (2009) Hidden killers: persistence of opportunistic fungal pathogens in the human host. Curr Opin Microbiol 12: 358-364.

Dantas S, Day A, Ikeh M, Kos I, Achan B, Quinn J (2015) Oxidative stress responses in the human fungal pathogen, Candida albicans. Biomolecules 5: 142-165.

Dujon B, Sherman D, Fischer G, Durrens P, Casaregola S, Lafontaine I, De Montigny J, Marck C, Neuvéglise C, Talla E, Goffard N, Frangeul L, Aigle M, Anthouard V, Babour A, Barbe V, Barnay S, Blanchin S, Beckerich JM, Beyne E, Bleykasten C, Boisramé A, Boyer J, Cattolico L, Confanioleri F, De Daruvar A, Despons L, Fabre E, Fairhead C, Ferry-Dumazet H, Groppi A, Hantraye F, Hennequin C, Jauniaux N, Joyet P, Kachouri R, Kerrest A, Koszul R, Lemaire M, Lesur I, Ma L, Muller H, Nicaud JM, Nikolski M, Oztas S, Ozier-Kalogeropoulos O, Pellenz S, Potier S, Richard GF, Straub ML, Suleau A, Swennen D, Tekaia F, Wésolowski-Louvel M, Westhof E, Wirth B, Zeniou-Meyer M, Zivanovic I, Bolotin-Fukuhara M, Thierry A, Bouchier C, Caudron B, Scarpelli C, Gaillardin C, Weissenbach J, Wincker P, Souciet JL (2004) Genome evolution in yeasts. Nature 430: 35-44.

Enjo F, Nosaka K, Ogata M, Iwashima A, Nishimura H (1997) Isolation and characterization of a thiamin transport gene, THI10, from Saccharomyces cerevisiae. I Biol Chem 272: 19165-19170.

Fernández-Arenas E, Cabezón V, Bermejo C, Arroyo J, Nombela C, Diez-Orejas R, Gil C (2007) Integrated proteomics and genomics strategies bring new insight into Candida albicans response upon macrophage interaction. Mol. Cell. Proteomics 6: 460-478.

Ferrari CKB, Souto PCS, França EL, Honorio-França AC (2011) Oxidative and nitrosative stress on phagocytes' function: from effective defense to immunity evasion mechanisms. Arch Immunol Ther Exp 59: 441-448.

Fink B, Laude K, Mccann L, Doughan A, Harrison D, Dikalov S (2004) Detection of intracellular superoxide formation in endothelial cells and intact tissues using dihydroethidium and an HPLC-based assay. Am J Physiol Cell Physiol 287: 895-902.

Fradin C, Kretschmar M, Nichterlein T, Gaillardin C, d'Enfert C, Hube B (2003) Stage- specific gene expression of Candida albicans in human blood. Mol Microbiol 47: 1523-1543.

Frohner IE, Bourgeois C, Yatsyk K, Majer O, Kuchler K (2009) Candida albicans cell surface superoxide dismutases degrade host-derived reactive oxygen species to escape innate immune surveillance. Mol Microbiol 71: 240-252.

Gangolf M, Czerniecki J, Radermecker M, Detry O, Nisolle M, Jouan C, Martin D, Chantraine F, Lakave B, Wins P, Grisar T, Bettendorff L (2010) Thiamine status in humans and content of phosphorylated thiamine derivatives in biopsies and cultured cells. PLoS One 5: 1-13.

Gasch AP, Spellman P, Kao C, Carmel-Harel O, Eisen M, Storz G, Botstein D (2000) Genomic expression programs in the response of yeast cells to environmental changes. Mol Biol Cell 11: 4241-4257.

Gibson GE, Blass JP (2007) Thiamine-dependent processes and treatment strategies in neurodegeneration. Antioxid Redox Signal 9: 16051619.
Gigliobianco T, Lakaye B, Wins P, El Moualij B, Zorzi W, Bettendorff L (2010) Adenosine thiamine triphosphate accumulates in Escherichia coli cells in response to specific conditions of metabolic stress. BMC Microbiol 10: 148-160.

Goyer A (2010) Thiamine in plants: aspects of its metabolism and functions. Phytochemistry 71: 1615-1624.

Iwashima A, Nose Y (1976) Regulation of thiamine transport in Saccharomyces cerevisiae. I Bacteriol 128: 855-857.

Jung IL, Kim IG (2003) Thiamine protects against paraquat-induced damage: scavenging activity of reactive oxygen species. Environ Toxicol Pharmacol 15: 19-26.

Kaur R, Domergue R, Zupancic ML, Cormack BP (2005) A yeast by any other name: Candida glabrata and its interaction with the host. Curr Opin Microbiol 8: 378-384. Kowalska E, Kozik A (2008) The genes and enzymes involved in the biosynthesis of thiamin and thiamin diphosphate in yeasts. Cell Mol Biol Lett 13: 271-282.

Kowalska E, Kujda M, Wolak N, Kozik A (2012) Altered expression and activities of enzymes involved in thiamine diphosphate biosynthesis in Saccharomyces cerevisiae under oxidative and osmotic stress. FEMS Yeast Res 12: 534-546.

Krcmery V, Barnes AJ (2002) Non-albicans Candida spp. causing fungaemia: pathogenicity and antifungal resistance. J Hosp Infect 50: $243-260$.

Lai RY, Huang S, Fenwick MK, Hazra A, Zhang Y, Rajashankar K, Philmus B, Kinsland C, Sanders JM, Ealick SE, Begley TP (2012) Thiamin pyrimidine biosynthesis in Candida albicans: A remarkable reaction between histidine and pyridoxal phosphate. J Am Chem Soc 134: 9157-9159.

Lakaye B, Wirtzfeld B, Wins P, Grisar T, Bettendorff L (2004) Thiamine triphosphate, a new signal required for optimal growth of Escherichia coli during amino acid starvation. J Biol Chem 279: 1714217147.

Livak KJ, Schmittgen TD (2001) Analysis of relative gene expression data using real-time quantitative PCR and the 2(-Delta Delta C(T)) Method. Methods 25: 402-408.

Lowry OH, Rosebrough NJ, Farr AL, Randall RJ (1951) Protein measurement with the Folin phenol reagent. J Biol Chem 193: 265-275.

Lukienko PI, Mel'nichenko NG, Zverinskii IV, Zabrodskaya SV (2000) Antioxidant properties of thiamine. Bull Exp Biol Med 130: 874-876.

Martchenko M, Alarco A, Harcus D, Whiteway M (2004) Superoxide dismutases in Candida albicans: transcriptional regulation and functional characterization of the hyphal-induced SOD5 gene. 15: 456467.

Miramón P, Dunker C, Windecker H, Bohovych IM, Brown AJP, Kurzai O, Hube B (2012) Cellular responses of Candida albicans to phagocytosis and the extracellular activities of neutrophils are critical to counteract carbohydrate starvation, oxidative and nitrosative stress. PLoS One 7: 1-14.

Mojzita D, Hohmann S (2006) Pdc2 coordinates expression of the THI regulon in the yeast Saccharomyces cerevisiae. Mol Genet Genomics 276: $147-161$.

Molero G, Díez-Orejas R, Navarro-García F, Monteoliva L, Pla J, Gil C, Sánchez-Pérez M, Nombela C (1998) Candida albicans: genetics, dimorphism and pathogenicity. Int Microbiol 1: 95-106.

Moran GP, Coleman DC, Sullivan DJ (2012) Candida albicans versus Candida dubliniensis: Why Is C. albicans More Pathogenic? Int I Microbiol 2012: 1-7.

Naglik JR, Challacombe SJ, Hube B (2003) Candida albicans secreted aspartyl proteinases in virulence and pathogenesis. 67: 400-428.

Nosaka K (2006) Recent progress in understanding thiamin biosynthesis and its genetic regulation in Saccharomyces cerevisiae. Appl Microbiol Biotechnol 72: 30-40.

Ozdemir MA, Akcakus M, Kurtoglu S, Gunes T, Torun YA (2002) TRMA syndrome (thiamine-responsive megaloblastic anemia): a case report and review of the literature. Pediatr Diabetes 3: 205-209.

Paul D, Chatterjee A, Begley TP, Ealick SE (2010) Domain organization in Candida glabrata THI6, a bifunctional enzyme required for thiamin biosynthesis in eukaryotes. Biochemistry 49: 9922-9934.

Pekovich SR, Martin PR, Singleton CK (1998) Thiamine deficiency decreases steady-state transketolase and pyruvate dehydrogenase but not alpha-ketoglutarate dehydrogenase mRNA levels in three human cell types. J Nutr 128: 683-687.

Pfaller MA, Diekema DJ (2007) Epidemiology of invasive candidiasis: A persistent public health problem. Clin Microbiol Rev 20: 133-163.

Ralser M, Wamelink MM, Kowald A, Gerisch B, Heeren G, Struys EA, Klipp E, Jakobs C, Breitenbach M, Lehrach H, Krobitsch S (2007) Dynamic rerouting of the carbohydrate flux is key to counteracting oxidative stress. J Biol 6: 1-18.

Ralser M, Wamelink MMC, Latkolik S, Jansen EEW, Lehrach H, Jakobs C (2009) Metabolic reconfiguration precedes transcriptional regulation in the antioxidant response. Nat Biotechnol 27: 604-605.

Rapala-Kozik M, Kowalska E, Ostrowska K (2008) Modulation of thiamine metabolism in Zea mays seedlings under conditions of abiotic stress. I Exp Bot 59: 4133-4143.

Rapala-Kozik M, Wolak N, Kujda M, Banas AK (2012) The upregulation of thiamine (vitamin B1) biosynthesis in Arabidopsis thaliana 
seedlings under salt and osmotic stress conditions is mediated by abscisic acid at the early stages of this stress response. BMC Plant Biol 12: 1-14.

Roetzer A, Gratz N, Kovarik P, Schüller C (2010) Autophagy supports Candida glabrata survival during phagocytosis. Cell Microbiol 12: 199-216.

Roetzer A, Gabaldón T, Schüller C (2011a) From Saccharomyces cerevisiae to Candida glabratain a few easy steps: important adaptations for an opportunistic pathogen. FEMS Microbiol Lett 314: 1-9.

Roetzer A, Klopf E, Gratz N, Marcet-Houben M, Hiller E, Rupp S, Gabaldon T, Kovarik P, Schüller C (2011b) Regulation of Candida glabrata oxidative stress resistance is adapted to host environment. FEBS Lett 585: 319-327.

Sauberlich HE (1967) Biochemical alterations in thiamine deficiency their interpretation. Am J Clin Nutr 20: 528-546.

Seider K, Gerwien F, Kasper L, Allert S, Brunke S, Jablonowski N, Schwarzmüller T, Barz D, Rupp S, Kuchler K, Hube B (2014) Immune evasion, stress resistance, and efficient nutrient acquisition are crucial for intracellular survival of Candida glabrata within macrophages. Eukaryot Cell 13: 170-183.

Selmecki A, Forche A, Berman J (2010) Genomic plasticity of the human fungal pathogen Candida albicans. Eukaryot. Cell 9: 991-1008.

Seneviratne CJ, Wang Y, Jin L, Abiko Y, Samaranayake LP (2010) Proteomics of drug resistance in Candida glabrata biofilms. Proteomics 10: 1444-1454.

Shi Q, Xu H, Kleinman WA, Gibson GE (2008) Novel functions of the alpha-ketoglutarate dehydrogenase complex may mediate diverse oxidant-induced changes in mitochondrial enzymes associated with Alzheimer's disease. Biochim Biophys Acta 1782: 229-238.

Singleton CK (1997) Identification and characterization of the thiamine transporter gene of Saccharomyces cerevisiae. Gene 199: 111-121.

Stepuro II, Oparin a Y, Stsiapura VI, Maskevich SA, Titov VY (2012) Oxidation of thiamine on reaction with nitrogen dioxide generated by ferric myoglobin and hemoglobin in the presence of nitrite and hydrogen peroxide. Biochem 77: 41-55.
Sudbery P, Gow N, Berman J (2004) The distinct morphogenic states of Candida albicans. Trends Microbiol 12: 317-324.

Sullivan DJ, Westerneng TJ, Haynes KA, Bennett DE, Coleman DC (1995) Candida dubliniensis sp. nov.: phenotypic and molecular characterization of a novel species associated with oral candidosis in HIV-infected individuals. Microbiology 141: 1507-1521.

Thornalley PJ (2005) The potential role of thiamine (vitamin B1) in diabetic complications. Curr Diabetes Rev 1: 287-298.

Tunc-Ozdemir M, Miller G, Song L, Kim J, Sodek A, Koussevitzky S, Misra AN, Mittler R, Shintani D (2009) Thiamin confers enhanced tolerance to oxidative stress in Arabidopsis. Plant Physiol 151: 421-432.

Vázquez-Torres A, Balish E (1997) Macrophages in resistance to candidiasis. Microbiol Mol Biol Rev 61: 170-192.

Wellington M, Dolan K, Krysan DJ (2009) Live Candida albicans suppresses production of reactive oxygen species in phagocytes. Infect Immun 77: 405-413.

Wojtaszek P (1997) Oxidative burst: an early plant response to pathogen infection. Biochem J 322: 681-692.

Wolak N, Kowalska E, Kozik A, Rapala-Kozik M (2014) Thiamine increases the resistance of baker's yeast Saccharomyces cerevisiae against oxidative, osmotic and thermal stress, through mechanisms partly independent of thiamine diphosphate-bound enzymes. FEMS Yeast Res 14: 1249-1262.

Wysong DR, Christin L, Sugar AM, Robbins PW, Diamond RD (1998) Cloning and sequencing of a Candida albicans catalase gene and effects of disruption of this gene. Infect Immun 66: 1953-1961.

Yin Z, Stead D, Walker J, Selway L, Smith DA, Brown AJP, Quinn J (2009) A proteomic analysis of the salt, cadmium and peroxide stress responses in Candida albicans and the role of the Hog1 stressactivated MAPK in regulating the stress-induced proteome. Proteomics 9: 4686-4703.

Zaoutis TE, Argon J, Chu J, Berlin JA, Walsh TJ, Feudtner C (2005) The epidemiology and attributable outcomes of candidemia in adults and children hospitalized in the United States: a propensity analysis. Clin Infect Dis 41: 1232-1239. 